\title{
Bosonization Approach to the One-Dimensional Kondo Lattice Model at Half Filling: Reexamination
}

\author{
Satoshi Fujimoto ${ }^{1}$ and Norio Kawakami ${ }^{2}$ \\ ${ }^{1}$ Department of Physics, Kyoto University, Kyoto 606, Japan \\ ${ }^{2}$ Department of Applied Physics, and Department of Material and Life Science, \\ Osaka University, Suita, Osaka 565, Japan
}

(Received )

\begin{abstract}
Low-energy properties of the one-dimensional Kondo lattice model are investigated by using bosonization and renormalization group methods. The formation of the spin and charge excitation gaps at half-filling is discussed in detail both for antiferromagnetic and ferromagnetic Kondo couplings. Also, the effect of the interaction between conduction electrons on the gap formation is studied both for the Kondo insulating phase and for the Haldane gap phase. It is found that while the repulsive interaction changes the dependence of the excitation gap on the Kondo exchange coupling, the attractive interaction can drive the Kondo (or Haldane) insulating phase to a completely different phase of massless metallic states.
\end{abstract}

KEYWORDS: Kondo lattice, heavy fermion, bosonizaton, spin gap

\section{$\S 1$. Introduction}

The Kondo lattice model is a fundamental model for heavy electron systems such as Cerium or Uranium compounds. One-dimensional (1D) version of the model has been studied ex-

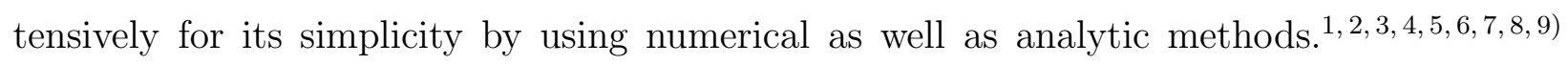
The model shows various phases according to the electron density and the strength of the Kondo coupling. At half-filling the Kondo insulating phase realizes, which has the excitation gaps both in the spin and charge sectors. Tomonaga-Luttinger liquid phase for small Kondo exchange couplings. .6.6) More recently, the effect of interaction between conduction electrons on these phases has been studied numerically (19) 
We have previously investigated this model by using bosonization method(6), which is a powerful tool to study low-energy properties of 1D quantum systems. In this method, one needs to linearize the dispersion of electrons at the first stage for deriving a low-energy effective hamiltonian. However, since localized spins have no dispersion, it is rather difficult to apply this method to the Kondo lattice model straightforwardly. In the previous paper, to bypass this difficulty we naively introduced a finite velocity for the excitation of localized spins in the microscopic model, and then performed bosonization.6) This prescription, however, may not give desirable functional forms of the spin and charge gaps for the original Kondo lattice model: the predicted gaps are not consistent with those obtained by numerical studies.2 In this paper, by starting with the original Kondo lattice model, we develop a low-energy effective theory improved in this respect, and reexamine the formation of the spin and charge gaps at half filling both for antiferromagnetic (AFM) and ferromagnetic (FM) Kondo couplings. Based upon this formulation, we also study how the short-range interaction between conduction electrons affects the spin/charge gap formation.

The organization of the paper is as follows. In $\S 2$ we introduce the bosonized effective hamiltonian for the 1D Kondo lattice model, and derive the scaling equations for various couplings which characterize the low-energy fixed point properties. In $\S 3$ we first discuss the case of the AFM Kondo coupling, and describe the formation of the spin and charge gaps in the Kondo insulating phase. The effect of interaction between conduction electrons on this phase is also investigated. It is shown that while the repulsive interaction destroys neither the spin nor charge gaps, the strong attractive interaction should change the Kondo insulating phase to a new gapless phase characterized by the Luther-Emery model and the $s=1 / 2$ spin chain model. In $\S 4$ the case of the FM Kondo coupling is also discussed in detail. Conclusion is given in $§ 5$.

\section{$\S 2$. Bosonization and scaling equations}

We first introduce the model hamiltonian for the 1D Kondo lattice, and apply the bosonization method to obtain the scaling equations which describe the fixed point properties. We consider the following hamiltonian,

$$
H=-t \sum_{i, \sigma} c_{\sigma i}^{\dagger} c_{\sigma i+1}+h . c .+U \sum_{i} c_{\uparrow i}^{\dagger} c_{\uparrow i} c_{\downarrow i}^{\dagger} c_{\downarrow i}+J_{K} \sum_{i, \alpha \beta} c_{\alpha i}^{\dagger} \frac{1}{2} \boldsymbol{\sigma}_{\alpha \beta} c_{\beta i} \cdot \boldsymbol{S}_{f i}
$$

where $c_{\sigma i}\left(c_{\sigma i}^{\dagger}\right)$ is an annihilation (creation) operator for conduction electrons at $i$-th site, and $\boldsymbol{S}_{f i}$ is an operator for localized $s=1 / 2$ spins. The second term is the on-site interaction between conduction electrons, and the last term is the Kondo exchange interaction. Note that we have not introduced the RKKY interaction here, which was added to the microscopic hamiltonian in our previous paper.6) In order to apply renormalization group methods to this 
model (2.1), we first bosonize the charge and spin degrees of freedom of conduction electrons and take their continuum limit with preserving full degrees of freedom of localized spins at each lattice site. Applying abelian 113 and non-abelian 12, 13) bosonizations to charge and spin degrees of freedom of conduction electrons respectively, we have the effective hamiltonian,

$$
\begin{aligned}
H= & H_{c}+H_{c-f}, \\
H_{c}= & \int \mathrm{d} x\left[\frac{v_{c}}{2 K_{c}}\left(\partial_{x} \phi_{c}(x)\right)^{2}+\frac{v_{c} K_{c}}{2} \Pi_{c}^{2}(x)\right]+\frac{U_{u m}}{\alpha} \int \mathrm{d} x \cos \left(\sqrt{8 \pi} \phi_{c}(x)+\delta x\right) \\
& +\int \mathrm{d} x v_{s}\left[\boldsymbol{J}_{c L}(x) \cdot \boldsymbol{J}_{c L}(x)+\boldsymbol{J}_{c R}(x) \cdot \boldsymbol{J}_{c R}(x)\right]-\lambda \int d x \boldsymbol{J}_{c L}(x) \cdot \boldsymbol{J}_{c R}(x), \\
H_{c-f}= & J_{K A} \int \mathrm{d} x\left[\boldsymbol{J}_{c L}(x)+\boldsymbol{J}_{c R}(x)\right] \cdot \boldsymbol{S}_{f}(x) \\
& +J_{K B} \int \frac{\mathrm{d} x}{\alpha}\left[e^{2 i k_{F} x} \frac{1}{2} \operatorname{tr}(g(x) \boldsymbol{\sigma}) e^{i \sqrt{2 \pi} \phi_{c}(x)}+\text { h.c. }\right] \cdot \boldsymbol{S}_{f}(x),
\end{aligned}
$$

where $\phi_{c}(x), \Pi_{c}(x)$ are boson fields for the charge degrees of freedom of conduction electrons and its conjugate momentum, and $\boldsymbol{J}_{c L, R}$ is a spin current operator which satisfies level-1 $\mathrm{SU}(2)$ Kac-Moody algebra, and $g(x)=c_{\alpha L}^{\dagger} c_{\beta R}(\alpha, \beta=\uparrow, \downarrow)$ is a fundamental representation of $\mathrm{SU}(2) . \boldsymbol{S}_{f}(x)=\sum_{i} f_{\alpha i}^{\dagger}\left(\boldsymbol{\sigma}_{\alpha \beta} / 2\right) f_{\beta i} \delta\left(x-x_{i}\right)$ is the spin density operator for localized spin. The $U_{u m^{-}}$and $\lambda$-terms are, respectively, the Umklapp and backward scattering terms of electronelectron interaction, and the parameter $\delta$ defined by $\delta=4 k_{\mathrm{F}}-2 \pi$ measures the deviation from the half-filling case. The forward scattering between conduction electrons is incorporated in the Tomonaga-Luttinger parameter $K_{c}$, via the initial value $K_{c}=1 / \sqrt{1+2 U / \pi v_{\mathrm{F}}}$.

In the microscopic model (2.1), the bare parameters should read $J_{K A}=J_{K B}=J_{K}$ and $U_{u m}=\lambda=U$, which are to be modified via the renormalization process. Note that if we regard the initial values of $K_{c}, U_{u m}$, and $\lambda$ as independent parameters, this effective hamiltonian describes the low-energy properties of a more general model than eq.(2.1), which has momentum-dependent interactions. We will also extend our arguments to such a general model in the subsequent sections. In what follows we mainly consider the half-filling case $\delta=0$. Thus we impose the commensurate condition, $2 k_{\mathrm{F}} a=\pi$ where $a$ is the lattice constant.

Now we derive the scaling equations for various coupling constants of the effective hamiltonian. For this purpose we utilize current algebra techniques for the operators. The charge field, $\phi_{c}$, satisfies the operator product expansion (OPE) of U(1) Gaussian model,14

$$
\begin{gathered}
e^{i \alpha \phi_{c}(x, \tau)} e^{-i \alpha \phi_{c}(0,0)} \sim \frac{i \alpha}{|z|^{\alpha^{2} K_{c} / 2 \pi-2}}\left(\frac{\partial_{z} \phi_{c}(0,0)}{\bar{z}}+\frac{\partial_{\bar{z}} \phi_{c}(0,0)}{z}\right) \\
+\frac{1}{|z|^{\alpha^{2} K_{c} / 2 \pi}}-\frac{\alpha^{2}}{|z|^{\alpha^{2} K_{c} / 2 \pi-2}} \partial_{z} \phi_{c}(0,0) \partial_{\bar{z}} \phi_{c}(0,0) \\
-\frac{\alpha^{2}}{2|z|^{\alpha^{2} K_{c} / 2 \pi}}\left(z^{2}\left(\partial_{z} \phi\right)^{2}+\bar{z}^{2}\left(\partial_{\bar{z}} \phi\right)^{2}\right)+\cdots
\end{gathered}
$$




$$
\partial_{z} \phi_{c}(x, \tau) e^{i \alpha \phi_{c}(0,0)} \sim \frac{i \alpha K_{c}}{8 \pi z} e^{i \alpha \phi_{c}(0,0)}+\cdots,
$$

with $z=x+i v_{c} \tau, \bar{z}=x-i v_{c} \tau . \quad \boldsymbol{J}_{c L, R}$ and $g(x)$ satisfy the OPE of level-1 SU(2) WessZumino-Witten model, 15, 16, 17)

$$
\begin{aligned}
J_{c L}^{a}(x, \tau) J_{c L}^{b}(0,0) & \sim \frac{\delta_{a b}}{z^{2}}+\frac{\varepsilon^{a b c}}{z} J_{c L}^{c}(0,0)+\cdots \\
J_{c L}^{a}(x, \tau) g(0,0) & \sim \frac{t^{a}}{z} g(0,0)+\cdots, \\
\operatorname{tr}\left[g(x, \tau) \sigma^{a}\right] \operatorname{tr}\left[g^{\dagger}(0,0) \sigma^{b}\right] & \sim \frac{\delta_{a b}}{|z|}+\left(\frac{z}{\bar{z}}\right)^{\frac{1}{2}} \varepsilon^{a b c} J_{c L}^{c}(0,0)+\left(\frac{\bar{z}}{z}\right)^{\frac{1}{2}} \varepsilon^{a b c} J_{c R}^{c}(0,0) \\
& +\frac{\delta_{a b}}{|z|}\left[z^{2} J_{c L}^{a}(0,0) J_{c L}^{a}(0,0)+\bar{z}^{2} J_{c R}^{a}(0,0) J_{c R}^{a}(0,0)\right]+\cdots
\end{aligned}
$$

with $z=x+i v_{s} \tau, \bar{z}=x-i v_{s} \tau$, and $t^{a}$, the generator of the $\mathrm{SU}(2)$ Lie algebra. We omit those terms which are irrelevant to the following arguments. Using these formulae, we can derive scaling equations,

$$
\begin{aligned}
\frac{d J_{K A}}{d \ln L} & =\frac{J_{K A}^{2}}{\pi v_{s}}+\frac{J_{K B}^{2}}{\pi v_{s}} f_{1}\left(\frac{v_{c}}{v_{s}}\right) \\
\frac{d J_{K B}}{d \ln L} & =\left(\frac{1-K_{c}}{2}+\frac{2 J_{K A}}{\pi v_{s}}+\frac{2 \lambda}{\pi v_{s}}+\frac{2 U_{u m}}{\pi v_{c}}\right) J_{K B}, \\
\frac{d \lambda}{d \ln L} & =-\frac{\lambda^{2}}{\pi v_{s}} \\
\frac{d U_{u m}}{d \ln L} & =\left(2-2 K_{c}\right) U_{u m}, \\
\frac{d K_{c}}{d \ln L} & =-K_{c}^{2} \frac{U_{u m}^{2}}{v_{c}^{2}}-K_{c}^{2} \frac{J_{K B}^{2}}{v_{s}^{2}} f_{2}\left(\frac{v_{c}}{v_{s}}\right),
\end{aligned}
$$

with

$$
\begin{aligned}
& f_{1}(u)=\int_{0}^{\pi} d \theta \frac{\sin \theta}{\left(\cos ^{2} \theta+u^{2} \sin ^{2} \theta\right)^{\frac{K_{c}}{2}}}, \\
& f_{2}(u)=\int_{0}^{\pi} d \theta \frac{1}{\left(\cos ^{2} \theta+u^{2} \sin ^{2} \theta\right)^{\frac{K_{c}}{2}-1}} .
\end{aligned}
$$

In the dilute limit of localized spins, the velocity of conduction electrons is not renormalized. In this case, if one neglects backward and Umklapp processes of electron-electron interaction, and expand the Luttinger liquid parameter up to the lowest order in the forward scattering, $K_{c} \sim 1-U / \pi$, one can reproduce the scaling equations for the single impurity Kondo problem in Luttinger liquids, which were obtained by g-ology methods.18) In the lattice system, the RKKY interaction between localized spins is generated by higher order processes of the Kondo exchange interaction. With the use of eq.(2.9), we obtain the RKKY interaction from the second order of $J_{K B}$,

$$
H_{R K K Y}=-J_{K B}^{2} \int d x \int d x^{\prime} f_{3}\left(\frac{v_{c}}{v_{s}}\right) \frac{\cos 2 k_{\mathrm{F}}\left(x-x^{\prime}\right)}{\left|x-x^{\prime}\right|} \boldsymbol{S}_{f}(x) \cdot \boldsymbol{S}_{f}\left(x^{\prime}\right),
$$


with

$$
f_{3}(u)=\int_{0}^{\pi} \mathrm{d} \theta \frac{1}{\left(\cos ^{2} \theta+u^{2} \sin ^{2} \theta\right)^{\frac{K_{c}}{2}}} .
$$

The predominant contribution comes from the nearest neighbor interaction. Since $2 k_{\mathrm{F}} a=\pi$, the RKKY interaction is antiferromagnetic for nearest neighbor sites. Thus we replace the RKKY interaction term approximately by

$$
H_{R K K Y}=J_{K B}^{2} \ln L f_{3}\left(\frac{v_{c}}{v_{s}}\right) \sum_{i} \boldsymbol{S}_{f i} \cdot \boldsymbol{S}_{f i+1} .
$$

Then the scaling equation for the RKKY interaction is given by

$$
\frac{d J_{R K K Y}}{d \ln L}=\frac{J_{K B}^{2}}{\pi v_{s}} f_{3}\left(\frac{v_{c}}{v_{s}}\right)
$$

Eqs. (2.10) (2.14) and (2.20) determine the low-energy properties of the model.

We note here that in our previous paper the RKKY interaction was naively introduced in the microscopic hamiltonian. In the following we will take into account that the RKKY term is a higher-order term generated by the Kondo interaction, and see what follows from this fact.

\section{§3. Antiferromagnetic Kondo coupling case}

We start our discussions with the case of AFM Kondo coupling $\left(J_{K}>0\right)$. In this case, from eqs.(2.10) and (2.20), we see that $J_{K A}$ and $J_{R K K Y}$ flow to the strong-coupling regime. Since initially $J_{K}>J_{R K K Y}=0$, the Kondo interaction $J_{K}$ is predominant over the RKKY interaction $J_{R K K Y}$. Thus low-energy properties at the fixed point are determined essentially by $J_{K}$. We have three different situations according to the interaction strength for conduction electrons: case $A$ : $U=0$ and $K_{c}(0)=1$, case $B: U>0$ and $K_{c}(0)<1$, and case $C$ : $U<0$ and $K_{c}(0)>1$.

\section{1 case $A: U=0$ and $K_{c}(0)=1$}

In the absence of interaction between conduction electrons, $U=0$, the scaling equations for the Kondo couplings are simplified as,

$$
\begin{aligned}
& \frac{d J_{K A}}{d \ln L}=\frac{J_{K A}^{2}}{\pi v_{s}}+\frac{J_{K B}^{2}}{\pi v_{s}} f_{1}\left(\frac{v_{c}}{v_{s}}\right), \\
& \frac{d J_{K B}}{d \ln L}=\frac{2 J_{K A} J_{K B}}{\pi v_{s}} .
\end{aligned}
$$

Thus $J_{K A}$ and $J_{K B}$ are marginally relevant, and produce spin and charge gaps. The fixed

point is the Kondo insulator as pointed out before. (6) If one neglects the renormalization of the velocity, the spin gap $\Delta_{s}$ and the charge gap $\Delta_{c}$ generated by $J_{K A}$ and $J_{K B}$ are given by

$$
\Delta_{s, c} \sim e^{-\frac{\pi v_{s}}{J_{K}}}
$$


for $J_{K} \ll v_{s}$. The $J_{K}$-dependence of the spin gap coincides with that obtained by Tsunetsugu et al.2.

\section{2 case $B: U>0$ and $K_{c}(0)<1$}

When the on-site interaction for conduction electrons is repulsive, the resulting $\lambda$-term (backward scattering) is marginally irrelevant, and negligible. $J_{K B}$ is a relevant interaction with scaling dimension $3 / 2+K_{c} / 2$ as seen from eq.(2.11). Since this dimension is larger than that of the Umklapp interaction, the Umklapp interaction $U_{u m}$ grows faster than $J_{K B}$ at half-filling, which may give rise to the Mott-Hubbard gap in the charge sector of conduction electrons. However if the initial value of $U_{u m}$ is much smaller than that of $J_{K B}, J_{K B}$-term may generate the charge gap before $U_{u m}$-term flows into the strong-coupling regime. Thus several different cross-over behaviors are expected to appear according to the initial values of parameters. In the following we show the expressions for spin and charge gaps in three limiting cases.

(i) $\frac{1-K_{c}(0)}{2} \gg \frac{2 J_{K A}(0)}{\pi v_{s}(0)}$ and $U_{u m}(0) / J_{K}(0) \gtrsim 1$.

The Mott-Hubbard gap due to $U_{u m}$-term opens prior to the spin gap formation due to $J_{K}$ in this case. After the charge gap formation, $K_{c}=0$, and eq.(2.11) becomes,

$$
\frac{d J_{K B}}{d \ln L}=\frac{J_{K B}}{2}
$$

Thus the spin gap for small $J_{K}$ is given by

$$
\Delta_{s} \sim J_{K}^{2} .
$$

The charge gap is given by the Mott-Hubbard gap of the 1D Hubbard model, if $J_{K}$ is sufficiently small compared to $U$.

(ii) $\frac{1-K_{c}(0)}{2} \gg \frac{2 J_{K A}(0)}{\pi v_{s}(0)}$ and $U_{u m}(0) / J_{K}(0) \ll 1$.

In this case, the charge gap generation due to $J_{K B}$-term is predominant over the Mott transition due to the Umklapp interaction between conduction electrons. Thus the spin and charge gap is given by

$$
\Delta_{s, c} \sim J_{K}^{\frac{2}{1-K_{c}}},
$$

for small $J_{K}$. The realization of this case requires a large forward scattering of electronelectron interaction and a small Umklapp interaction at the initial stage of the renormalization process. However, in the microscopic model given by eq. 2.1), these parameters are initially equal to each other. Thus in order to actually observe this behavior, we should consider a more general model such as the g-ology model which has different coupling constants of electron-electron interaction depending on transfered momentum.19) 
(iii) $\frac{1-K_{c}(0)}{2} \ll \frac{2 J_{K A}(0)}{\pi v_{s}(0)}$.

In this case, the first term of the right hand side of eq.(2.11) is negligible. Thus the spin gap has the same $J_{K}$-dependence as the case of $K_{c}=1$ ( $U=0$ case).

$$
\Delta_{s} \sim e^{-\frac{\pi v_{s}}{J_{K}}}
$$

The charge gap in this case is generated by $U_{u m}$-term or $J_{K B^{-}}$term depending on the initial value of these couplings.

It is to be noted here that the above different behaviors observed in the gap formation do not imply that there appear new different universality classes: the system still belongs to the same universality of the Kondo insulating phase as far as $U \geq 0$, and cross-over behaviors rather than phase boundaries are seen when the initial values of bare parameters are changed.

We would like to compare the above results with those obtained by Shibata et al. (19). They calculated numerically the spin gap of the model given by eq.(2.1). They concluded that the spin gap is given by $\Delta_{s} \sim \exp \left(-\pi v_{s} / \alpha J_{K}\right)$ for small $J_{K}$ and any $U$, where a parameter $\alpha$ depends on $U$. Their conclusion does not seem to coincide with our results in the case (i) shown above. Actually, the parameter region where their calculation was performed corresponds to the case (iii) or the case where $\left(1-K_{c}(0)\right) / 2$ is of the same order as $J_{K A}(0) / \pi v_{s}(0)$. We have solved numerically the scaling equations when $\left(1-K_{c}(0)\right) / 2$ is of the same order as $J_{K A}(0) / \pi v_{s}(0)$, and deduced the functional dependence of the spin gap on $J_{K}$. We found that the results fit in with the exponential function fairly well. Thus our results are consistent with those of Shibata et al. We think that the value of $J_{K}$ they used may not be small enough to observe the power-law dependence of eq.(3.5).

\section{3 case $C: U<0$ and $K_{c}(0)>1$}

In the attractive case of $U$, the Umklapp term is irrelevant, and can be discarded. However since $\lambda<0$, the $\lambda$-term is marginally relevant. The $J_{K B}$-term may be relevant or irrelevant depending on $K_{c}(0)$ and $J_{K}$. Therefore we have three different behaviors as shown below.

(i) $J_{K B}$ predominates over $J_{K A}$ and $\lambda$.

If $|U|$ is sufficiently small compared to $J_{K}$, and $K_{c}(0)$ is not so large, the $J_{K B^{-}}$term is still relevant. The spin and charge gaps are generated by the $J_{K A^{-}}$and $J_{K B^{-}}$terms. For small $J_{K}$, the gaps are given by

$$
\Delta_{s, c} \sim e^{-\frac{\pi v_{s}}{J_{K}}}
$$

Note that the universality class still belongs to that of the Kondo insulator.

(ii) $J_{K A}$ predominates over $J_{K B}$ and $\lambda$. 
If $K_{c}(0)$ is sufficiently large, the $J_{K B}$-term becomes irrelevant. Moreover if $|\lambda(0)|$ is smaller than $J_{K}, J_{K A}$ grows to a strong-coupling value before $\lambda$ flows into the strong-coupling regime. In this case, the $J_{K A}$-term generates the spin gap given by

$$
\Delta_{s} \sim e^{-\frac{\pi v_{s}}{J_{K}}} .
$$

The charge sector is massless. Thus the fixed point of the charge degrees of freedom is the Tomonaga-Luttinger liquids described by $c=1 \mathrm{U}(1)$ Gaussian model. This would bring about a new phase driven by the interaction. However, we recall that for the microscopic model given by eq.(2.1), we can not control the parameters $K_{c}(0)$ and $\lambda(0)$ independently. Thus it is not obvious whether this phase may realize for the model (2.1). If one considers the model in which the strength of the forward scattering of electron-electron interaction which controls $K_{c}(0)$ and the backward scattering $\lambda(0)$ are independent parameters, one can surely find this phase.

(iii) $\lambda$ predominates over $J_{K A}$ and $J_{K B}$.

We now discuss the case where $|U|$ is sufficiently large and $|U| \gg J_{K}$. Then the $J_{K B^{-}}$ term is irrelevant, and thus the charge sector becomes massless, forming the TomonagaLuttinger liquid described by $c=1 \mathrm{U}(1)$ Gaussian model. On the other hand, the spin gap generation occurs due to the $\lambda$-term (backward scattering term) prior to that due to the $J_{K A}$-term. This state obtained for conduction electrons is nothing but a metallic state with the spin gap which is often referred to as the Luther-Emery class. Localized spins are now completely decoupled from conduction electrons, making a massless mode classified as SU(2) Tomonaga-Luttinger liquid described by $c=1$ level-1 SU(2) Wess-Zumino-Witten model. Therefore it turns out that a sufficiently large attractive interaction should drive the Kondo insulator to a completely different phase, in which both of the spin and charge sectors are massless: conduction electrons are in the Luther-Emery phase and localized spins are in SU(2) Tomonaga-Luttinger liquid. Recently Shibata et al. have also found this massless phase for large $|U|$ by using the density matrix renormalizatoin group method.20)

\section{$\S 4$. Ferromagnetic Kondo coupling case}

We now move to the discussions for the case of FM Kondo couplings. It is known that the spin gap a la Haldane opens at half filling in this case 2,21) We now explore how the formation of the spin and charge gaps is affected by the electron-electron interaction for conduction electrons.

\section{$4.1 U=0$ and $K_{c}(0)=1$}

In this case, as is seen from eqs.(2.10) and (2.11), the $J_{K A^{-}}$and $J_{K B^{-}}$-terms are marginally irrelevant, and these couplings scale to zero. In the process of the renormalization, $J_{R K K Y}$ 
becomes larger than $\left|J_{K A}\right|$ and $\left|J_{K B}\right|$. Thus we can not discard the RKKY interaction compared to the Kondo coupling. The induced RKKY interaction gives rise to a dispersion of localized spins, and thus changes the scaling equations obtained in $\S 2$. This point is quite contrasted to the antiferromagnetic case discussed above, where the $J_{K A^{-}}$and $J_{K B^{-}}$terms are marginally relevant. For $J_{R K K Y} \gg\left|J_{K A}\right|,\left|J_{K B}\right|$, we can bosonize localized spins in the continuum limit, and treat the Kondo coupling perturbatively. Then the field theoretical limit of the RKKY interaction is

$$
H_{R K K Y}=\int \mathrm{d} x v_{f}\left[\boldsymbol{J}_{f L}(x) \cdot \boldsymbol{J}_{f L}(x)+\boldsymbol{J}_{f R}(x) \cdot \boldsymbol{J}_{f R}(x)\right]-\lambda_{f} \int \mathrm{d} x \boldsymbol{J}_{f L}(x) \cdot \boldsymbol{J}_{f R}(x),
$$

where $\boldsymbol{J}_{f L, R}$ satisfy level-1 SU(2) Kac-Moody algebra. $\lambda_{f}>0$ is a coupling constant for the marginally irrelevant operator which exists because of chiral $\mathrm{SU}(2) \times \mathrm{SU}(2)$ symmetry. This is level-1 SU(2) Wess-Zumino-Witten model with marginally irrelevant interaction. We now incorporate this RKKY term into a non-perturbed part of the hamiltonian. Then the bosonized expression of the Kondo exchange interaction is altered to

$$
\begin{aligned}
H_{c-f}= & J_{K A} \int \mathrm{d} x\left(\boldsymbol{J}_{c L}(x)+\boldsymbol{J}_{c R}(x)\right) \cdot\left(\boldsymbol{J}_{f L}(x)+\boldsymbol{J}_{f R}(x)\right) \\
& +J_{K B} \int \mathrm{d} x\left[\operatorname{tr}\left(g(x) \frac{\boldsymbol{\sigma}}{2}\right) \cdot \operatorname{tr}\left(g_{f}(x) \frac{\boldsymbol{\sigma}}{2}\right) e^{i \sqrt{2 \pi} \phi_{c}(x)}+h . c .\right],
\end{aligned}
$$

where $g_{f}(x)=f_{\alpha L}^{\dagger}(x) f_{\beta R}(x)(\alpha, \beta=\uparrow, \downarrow)$. The scaling dimension of the second term of eq. (4.2) is now $1+K_{c} / 2$. Note that $K_{c}$ is renormalized by the $J_{K B}$-term and should be smaller than 1 . This term is relevant and generates the spin and charge gaps. The low-energy fixed point is nothing but the Haldane gap phase 2 Although the spin and charge gaps are given by the power-law function of $J_{K B}$, we cannot write down their expressions explicitly in terms of bare coupling $J_{K}$, because $J_{K B}$ in eq.(4.2) is renormalized, being different from bare $J_{K}$.

\section{2 $U>0$ and $K_{c}(0)<1$}

If we introduce the repulsive interaction for conduction electrons, $J_{K B}$ becomes relevant, as is seen from eq.(2.11). Thus the spin and charge gaps open as discussed in §3.2. The spin gap is given by eq.(3.5) or (3.6). The universality class is still characterized by the insulating phase with the Haldane-gap.

\section{$4.3 U<0$ and $K_{c}(0)>1$}

In the case of attractive interaction, the $J_{K A^{-}}$and $J_{K B^{-}}$-terms of eq.(2.4) and the Umklapp interaction $U_{u m}$ are irrelevant. Thus we should take into account the RKKY interaction again as in the case of $U=0$. We bosonize localized spin and use eq.(4.2) as the effective 
Kondo exchange interaction. Then the scaling equation for $J_{K B}$ is changed to

$$
\frac{d J_{K B}}{d \ln L}=\left(1-\frac{K_{c}}{2}+\frac{2 J_{K A}}{\pi v_{s}}+\frac{2 \lambda}{\pi v_{s}}+\frac{2 \lambda_{f}}{\pi v_{f}}+\frac{2 U_{u m}}{\pi v_{c}}\right) J_{K B} .
$$

The $J_{K B^{-}}$-term may be relevant or irrelevant depending on the initial values of $\lambda=\lambda_{0}$ and $K_{c}=K_{c 0}$, which are renormalized to the values different from $U$ and $K_{c}(0)$. Moreover, as is discussed in $\S 3.3$, the $\lambda$-term flows to a strong-coupling regime. Thus we have two possibilities: (i) $J_{K B}$ predominates over $\lambda$, (ii) $\lambda$ predominates over $J_{K B}$.

(i) $J_{K B}$ predominates over $\lambda$.

If $\left|\lambda_{0}\right|$ is sufficiently small, and $K_{c 0}$ is not so large, $J_{K B}$ may be relevant. Since $J_{K B}$ grows faster than $\lambda$, the $J_{K B}$-term generates spin and charge gaps prior to the spin gap formation due to the $\lambda$-term provided that the initial value of $J_{K B}$ is not much smaller than $\lambda_{0}$. The fixed point is the same class as the Haldane gap phase.

(ii) $\lambda$ predominates over $J_{K B}$.

For sufficiently large negative $U, \lambda$ flows to a strong-coupling regime, $\lambda \rightarrow-\infty$, faster than $J_{K B}$. Then, the right hand side of eq.(4.3) becomes positive. The $J_{K B}$-term is irrelevant in this case. Thus the charge sector of conduction electrons is massless, and described by $c=1$ U(1) Gaussian model, i.e. the Tomonaga-Luttinger liquids, and the spin gap of conduction electrons opens due to the $\lambda$-term, as has been discussed for the AFM case. Therefore, even for the FM case, a sufficiently strong attractive interaction brings the system to a massless phase with conduction electrons in the Luther-Emery phase and localized spins in the $\mathrm{SU}(2)$ Tomonaga-Luttinger phase.

\section{$\S 5$. Conclusion}

We have studied the 1D Kondo lattice model by means of bosonization techniques, and discussed how the mass gap is generated at half filling. It has been confirmed that the spin and charge gaps are indeed generated both for the AFM and FM Kondo couplings, realizing the Kondo insulating phase and the Haldane-gap insulating phase. By taking into account that the RKKY interaction is a higher order term induced by the Kondo coupling, we have shown that the generation of the spin gap occurs in different manners for the AFM and FM cases. This is one of the central points to be claimed in the present paper, which was not considered in our previous paper 6 The results thus obtained for the spin gap are consistent with the previous numerical studies, and may explain why the functional dependence of the spin gap on $J_{K}$ can differ between the AFM and FM couplings.

We have also investigated how the short-range interaction between conduction electrons affects on the formation of these excitation gaps. It has been shown that although the 
repulsive interaction changes the functional dependence of spin and charge gaps on the Kondo coupling, the universality class is still characterized by that for $U=0$. On the other hand, a sufficiently strong attractive interaction can destroy the spin and charge gaps, resulting in a completely different class of massless liquids consisting of the Luther-Emery state and the SU(2) Tomonaga-Luttinger liquid state.

We have seen that the spin gap formation can be described rather well in the present approach. As for the charge gap, however, we have not been able to completely resolve the discrepancy between the present results and the numerical results, although the qualitative agreement was already established concerning whether the mass is generated or not. For example, the exponential dependence of the charge gap on $J_{K}$ obtained for the AFM case

with $U=0$ does not seem to fit in with the $J_{K}$-linear dependence deduced numerically. (4) To resolve this problem is one of the main issues to be addressed in the future work.

Finally we wish to note that the present results are applicable not only to the model with the Hubbard-like on-site interaction but also to more general models with momentumdependent electron-electron interaction. For such general models, one can find the metallic phase with spin gap predicted in $\S 3$ for the AFM Kondo model with attractive electronelectron interaction.

\section{Acknowledgement}

One of the authors (S.F.) thanks N. Shibata for valuable discussions. This work was partly supported by a Grant-in-Aid from the Ministry of Education, Science and Culture.

1) R. M. Fye and D. J. Scalapino, Phys. Rev. Lett. 65 (1990) 3177.

2) H. Tsunetsugu, Y. Hatsugai, K. Ueda, and M. Sigrist, Phys. Rev. B46 (1992) 3175.

3) M. Troyer and D. Würtz, Phys. Rev. B47 (1993) 2886.

4) K. Ueda, T. Nishino, and H. Tsunetsugu, Phys. Rev. B50 (1994) 612.

5) A. M. Tsvelik, Phys. Rev. Lett. 72 (1994) 1048.

6) S. Fujimoto and N. Kawakami, J. Phys. Soc. Jpn. 63 (1994) 4322.

7) O. Zachar, S. A. Kivelson, and V. J. Emery, Phys. Rev. Lett. 77 (1996) 1342.

8) S. Moukouri and L. G. Caron, Phys. Rev. B52 (1995) 15723; ibid 54 (1996) 12212.

9) S. White and I. Affleck, Phys. Rev. B54 (1996) 9862.

10) N. Shibata, T. Nishino, and K. Ueda, Phys. Rev. B53 (1996) 8828.

11) F. D. M. Haldane, J. Phys. C14 (1981) 2585; Phys. Rev. Lett. 47 (1981) 1840; ibid 45 (1980) 1358.

12) E. Witten, Comm. Math. Phys. 92 (1984) 455.

13) I. Affleck, Nucl. Phys. B265, 409 (1986); Fields, Strings and Critical Phenomena, ed. E. Brezin and J. Zinn-Justin (North-Holland, Amsterdam, 1990).

14) L. P. Kadanoff, Ann. Phys. 120, 39 (1979); L. P. Kadanoff and A. C. Brown, ibid. 121, 318 (1979.) 
15) V. Knizhnik and A. Zamolodchikov, Nucl. Phys. B247 (1984) 83.

16) P. Bouwknegt, A. W. W. Ludwig, and K. Schoutens, Phys. Lett. B338, 448 (1994).

17) D. Bernard, V. Pasquier, and D. Serban, Nucl. Phys. B428, 612 (1994).

18) A. Furusaki and N. Nagaosa, Phys. Rev. Lett. 72 (1994) 892.

19) J. Sólyom, Adv. Phys. 28 (1979) 201.

20) N. Shibata, private communication.

21) F. D. M. Haldane, Phys. Rev. Lett. 50 (1983) 1153; Phys. Lett. 93A (1983) 464. 